\title{
Numerical simulation of soil-structure interaction in framed and shear-wall structures
}

\begin{abstract}
This paper deals with the modeling of the plane frame structure-foundation-soil system. The superstructure along with the foundation beam is idealized as beam bending elements. The soil medium near the foundation beam with stress concentrated is idealized by isoparametric finite elements, and infinite elements are used to represent the far field of the soil media. This paper presents the modeling of shear wall structure-foundation and soil system using the optimal membrane triangular, super and conventional finite elements. Particularly, an alternative formulation is presented for the optimal triangular elements aimed at reducing the programming effort and computational cost. The proposed model is applied to a plane framecombined footing-soil system. It is shown that the total settlement obtained from the nonlinear interactive analysis is about 1.3 to 1.4 times that of the non-interactive analysis. Furthermore, the proposed model was found to be efficient in simulating the shear wallfoundation-soil system, being able to yield results that are similar to those obtained by the conventional finite element method.
\end{abstract}

Keyword: Triangular element; Shear wall structure; Super element; Drilling degree of freedom 